\title{
6-(2-Alkylimidazol-1-yl)purines Undergo Regiospecific Glycosylation at N9
}

Minghong Zhong, Ireneusz Nowak, and Morris J. Robins*

Department of Chemistry and Biochemistry, Brigham Young University, Provo, Utah 84602-5700

morris_robins@byu.edu

\section{Supporting Information}

Pages S2-S7: Experimental procedures and characterization data

Pages S8-S13: ${ }^{13} \mathrm{C}$ NMR spectra of 3a-d, 5a-d, and $\mathbf{6 a}-\mathbf{d}$ 


\section{Experimental Section}

General: Uncorrected melting points were determined with a Hoover Capillary apparatus. ${ }^{1} \mathrm{H}(500$ $\mathrm{MHz})$ and ${ }^{13} \mathrm{C}(125 \mathrm{MHz}) \mathrm{NMR}$ spectra were recorded in $\mathrm{CDCl}_{3}$ unless otherwise noted. Reagent grade chemicals were used. Acetonitrile (ACN) was stored over $\mathrm{CaH}_{2}$ and 1,2-dichloroethane (DCE) was stored over molecular sieves.

Synthesis of 6-(imidazol-1-yl)purine (1a) and 6-(2-propylimidazol-1-yl)purine (1b).

General Procedure 1: A suspension of 2',3',5'-tri- $O$-acetylinosine (1.58 g, $4.0 \mathrm{mmol}), 2$ propylimidazole $^{1}(1.60 \mathrm{~g}, 14.4 \mathrm{mmol}), \mathrm{PPh}_{3}(1.60 \mathrm{~g}, 14.4 \mathrm{mmol}), \mathrm{I}_{2}(2.14 \mathrm{~g}, 8.3 \mathrm{mmol})$, and $\mathrm{EtN}(\mathrm{iPr})_{2}(3.6 \mathrm{~mL}, 2.67 \mathrm{~g}, 20.2 \mathrm{mmol})$ in dried toluene $(40 \mathrm{~mL})$ was stirred at $95{ }^{\circ} \mathrm{C}$ for $4 \mathrm{~h}$. Volatiles were evaporated in vacuo and the residue was extracted with boiling EtOAc. Volatiles were evaporated from the combined extracts and the residue was chromatographed $\left(\mathrm{MeOH} / \mathrm{CH}_{2} \mathrm{Cl}_{2}\right.$, 1:40) to give a solid contaminated with $\mathrm{Ph}_{3} \mathrm{PO}$. This material was dissolved in $\mathrm{AcOH}(160 \mathrm{~mL})$, and $\mathrm{AcCl}(2.2 \mathrm{~mL}, 2.43 \mathrm{~g}, 31 \mathrm{mmol})$ was added. The solution was stirred at $65{ }^{\circ} \mathrm{C}$ overnight, and volatiles were evaporated in vacuo. The residue was dissolved in $\mathrm{CH}_{2} \mathrm{Cl}_{2}$ and extracted with $0.1 \mathrm{~N}$ $\mathrm{NaOH}$. The aqueous layer was washed with $\mathrm{CH}_{2} \mathrm{Cl}_{2}$, and the product was precipitated with $\mathrm{CO}_{2}$. The solid was filtered and thoroughly washed $\left(\mathrm{H}_{2} \mathrm{O}\right)$ to give a solid $(0.66 \mathrm{~g}, 72 \%)$. This material was dissolved in $\mathrm{MeOH}$ and decolorized with charcoal. Recrystallization $(\mathrm{MeOH})$ gave $\mathbf{1 b}$ as a colorless solid: $\operatorname{mp} 242.5-243.5^{\circ} \mathrm{C}$; UV (MeOH) $\max 278 \mathrm{~nm}$ (ع 13700$)$, $\min 235 \mathrm{~nm}$ (ع 5000); ${ }^{1} \mathrm{H}$ NMR $\left(\mathrm{DMSO}-d_{6}\right) \delta 0.93(\mathrm{t}, J=7.3 \mathrm{~Hz}, 3 \mathrm{H}), 1.79-1.88(\mathrm{~m}, 2 \mathrm{H}), 3.18(\mathrm{t}, J=7.3 \mathrm{~Hz}, 2 \mathrm{H}), 7.07(\mathrm{~d}, J=1.5$ $\mathrm{Hz}, 1 \mathrm{H}), 8.36,8.69,8.86(3 \times \mathrm{s}, 3 \times 1 \mathrm{H}), 13.90($ br s, $1 \mathrm{H}) ;{ }^{13} \mathrm{C}$ NMR $\delta$ 14.5, 21.5, 32.2, 121.4, 123.6, 128.5, 145.8, 146.9, 149.8, 152.0, 155.5; HMRS Calcd for $\mathrm{C}_{11} \mathrm{H}_{12} \mathrm{~N}_{6}$ : 228.1123, Found: 228.1109; Anal. Calcd for $\mathrm{C}_{11} \mathrm{H}_{12} \mathrm{~N}_{6}$ : C, 57.88; H, 5.30; N, 36.82. Found: C, 58.09; H, 5.19; N, 37.00 .

6-(Imidazol-1-yl)purine ${ }^{2}$ (1a). Synthesized by general procedure 1: UV (MeOH) max 278 $\mathrm{nm}\left(\varepsilon 15\right.$ 100), min $234 \mathrm{~nm}$ ( $\varepsilon$ 3300); ${ }^{1} \mathrm{H}$ NMR (DMSO- $\left.d_{6}\right) \delta$ 7.26-7.27 (m, 1H), 8.42-8.44 (m, $1 \mathrm{H}), 8.72,8.82(2 \times \mathrm{s}, 2 \times 1 \mathrm{H}) ; 9.09-9.10(\mathrm{~m}, 1 \mathrm{H}), 13.90($ br s, $1 \mathrm{H})$.

\footnotetext{
${ }^{1}$ The 2-alkylimidazoles were prepared by reported procedures: (a) Katritzky, A. R.; Rewcastle, G. W.; Fan, W. Q. J. Org. Chem. 1988, 53, 5685-5689. (b) Arduengo, A. J., III; Gentry, F. P., Jr.; Taverkere, P. K.; Simmons, H. E., III U.S. Patent 6,177,575; 2001; Chem. Abst. 2001, 134, 115958.

${ }^{2}$ Estep, K. G.; Josef, K. A.; Bacon, E. R.; Carabateas, P. M.; Rumney, S., IV; Pilling, G. M.; Krafte, D. S.; Volberg, W. A.; Dillon, K.; Dugrenier, N.; Briggs, G. M.; Canniff, P. C.; Gorczyca, W. P.; Stankus, G. P.; Ezrin, A. M. J. Med. Chem. 1995, 38, 2582-2595.
} 


\section{Coupling of 6-(imidazol-1-yl)purine (1a) with 1- $O$-acetyl-2,3,5-tri- $O$-benzoyl- $\beta$-D-}

ribofuranose (2a). Method A: Purified 1a (52 $\mathrm{mg}, 0.28 \mathrm{mmol})$ was suspended in a solution of $2 \mathbf{a}$ (160 mg, $0.32 \mathrm{mmol})$ in dried $\mathrm{CH}_{3} \mathrm{CN}$ (10 mL). $\mathrm{SnCl}_{4}(0.10 \mathrm{~mL}, 0.22 \mathrm{~g}, 0.85 \mathrm{mmol})$ was added, and the mixture became a clear solution. The solution was stirred at ambient temperature for $4 \mathrm{~h}$. Solid $\mathrm{NaHCO}_{3}(800 \mathrm{mg}, 9.5 \mathrm{mmol})$ and water $(0.1 \mathrm{~mL}, 5.6 \mathrm{mmol})$ were added, and the suspension was stirred for $1 \mathrm{~h}$. The clear solution layer was separated, and the residue was extracted with $\mathrm{CH}_{3} \mathrm{CN}$. Volatiles were evaporated from the combined solution layer and extracts. The residue was chromatographed $\left(\mathrm{MeOH} / \mathrm{CH}_{2} \mathrm{Cl}_{2}, 1: 90 \rightarrow 1: 15\right)$ to give the coupling product 3a (179 mg, quant.): ${ }^{1} \mathrm{H}$ NMR $\delta 4.72(\mathrm{dd}, J=4.3,12.2 \mathrm{~Hz}, 1 \mathrm{H}), 4.88$ (br s, 1H), 4.95 (dd, $\left.J=3.0,12.2 \mathrm{~Hz}, 1 \mathrm{H}\right), 6.29$ (t, $J$ $=5.2 \mathrm{~Hz}, 1 \mathrm{H}), 6.47-6.50(\mathrm{~m}, 2 \mathrm{H}), 7.24(\mathrm{~s}, 1 \mathrm{H}), 7.35-8.07(\mathrm{~m}, 15 \mathrm{H}), 8.28,8.35,8.65,9.13(4 \times \mathrm{s}, 4$ $\times 1 \mathrm{H}) ;{ }^{13} \mathrm{C}$ NMR $\delta 62.3,70.3,72.9,79.9,86.4,116.3,122.0,127.2,127.5,128.2,128.7,128.8$, 129.7, 132.4, 132.8, 132.9, 136.6, 142.2, 144.9, 151.5, 152.1, 164.1, 164.3, 165.0; HMRS Calcd for $\mathrm{C}_{34} \mathrm{H}_{26} \mathrm{~N}_{6} \mathrm{O}_{7} \mathrm{Na}$ : 653.1761, Found: 653.1749.

Method B: N,O-Bis(trimethylsilyl)acetamide (BSA) $(0.26 \mathrm{~mL}, 0.21 \mathrm{~g}, 1.0 \mathrm{mmol})$ was added to a suspension of $1 \mathbf{a}(93 \mathrm{mg}, 0.5 \mathrm{mmol})$ in dried DCE $(10 \mathrm{~mL})$. The mixture was stirred at $80{ }^{\circ} \mathrm{C}$, and a clear solution resulted in $\sim 2 \mathrm{~h}$. Volatiles were evaporated in vacuo, the residue was dissolved in dried toluene $(5 \mathrm{~mL})$, and $\mathbf{2 a}(311 \mathrm{mg}, 0.6 \mathrm{mmol})$ and TMSOTf $(0.13 \mathrm{~mL}, 0.31 \mathrm{~g}, 0.8 \mathrm{mmol})$ were added to the stirred solution. Stirring was continued for $2 \mathrm{~h}$ at $80{ }^{\circ} \mathrm{C}$ and the solution was allowed to cool. Volatiles were evaporated, and the residue was stirred with $\mathrm{NaHCO} / \mathrm{H}_{2} \mathrm{O} / / \mathrm{CH}_{2} \mathrm{Cl}_{2}$ $(130 \mathrm{~mL} / / 60 \mathrm{~mL})$ overnight. The organic phase was dried $\left(\mathrm{Na}_{2} \mathrm{SO}_{4}\right)$, and volatiles were evaporated. Chromatography of the residue $\left(\mathrm{MeOH} / \mathrm{CH}_{2} \mathrm{Cl}_{2}, 1: 30 \rightarrow 1: 12\right)$ gave 4 (265 mg, 84\%) (two diastereomers): ${ }^{1} \mathrm{H}$ NMR $\delta 4.52$ (and 4.58) (dd, $\left.J=3.0,12.2 \mathrm{~Hz}, 1 \mathrm{H}\right), 4.70$ (br s, 1H), 4.83-4.86 (m, $1 \mathrm{H}), 5.48$ (and 5.62) $(\mathrm{d}, J=7.4 \mathrm{~Hz}, 1 \mathrm{H}), 7.30-7.43(\mathrm{~m}, 6 \mathrm{H}), 7.54-7.60(\mathrm{~m}, 3 \mathrm{H}), 7.77-8.00(\mathrm{~m}$, $14 \mathrm{H}), 8.28(\mathrm{~d}, J=7.7 \mathrm{~Hz}, 1 \mathrm{H}), 8.31,8.52(2 \times \mathrm{s}, 2 \times 1 \mathrm{H}) ;$ HMRS Calcd for $\mathrm{C}_{34} \mathrm{H}_{28} \mathrm{~N}_{6} \mathrm{O}_{8} \mathrm{Na}$ : 671.1886, Found: 671.1882.

The reaction was repeated with 1a $(186 \mathrm{mg}, 1.0 \mathrm{mmol})$. Volatiles were evaporated in vacuo, and the residue was chromatographed $\left(\mathrm{MeOH} / \mathrm{CH}_{2} \mathrm{Cl}_{2}, 1: 30\right)$ to give a protonated imidazolium salt (778 mg): ${ }^{1} \mathrm{H}$ NMR $\delta$ 4.76-4.80 (m, 1H), 5.00-5.09 (m 2H), 5.85 (br s, 1H), 5.99 (t, $J=5.4 \mathrm{~Hz}$, 1H), 6.07-6.10 (m, 1H), $6.81(\mathrm{~d}, J=3.6 \mathrm{~Hz}, 1 \mathrm{H}), 7.22-7.61(\mathrm{~m}, 9 \mathrm{H}), 7.89-8.03(\mathrm{~m}, 6 \mathrm{H}), 8.12(\mathrm{~d}, J$ $=1.5 \mathrm{~Hz}, 1 \mathrm{H}), 8.23,8.64,8.78,10.46(4 \times \mathrm{s}, 4 \times 1 \mathrm{H}) ; \mathrm{HMRS}$ Calcd for $\mathrm{C}_{34} \mathrm{H}_{26} \mathrm{~N}_{6} \mathrm{O}_{7} \mathrm{Na}: 653.1761$, Found: 653.1742. This salt was stirred with $\mathrm{NaHCO}_{3} / \mathrm{H}_{2} \mathrm{O} / / \mathrm{CH}_{2} \mathrm{Cl}_{2}$ to give $4(511 \mathrm{mg}, 81 \%)$. 


\section{Coupling of 6-(2-propylimidazol-1-yl)purine (1b) with glycosyl donors 2. General}

Procedure 2: $\mathrm{SnCl}_{4}(0.10 \mathrm{~mL}, 0.22 \mathrm{~g}, 0.85 \mathrm{mmol})$ was added dropwise to a stirred suspension of $\mathbf{1 b}$ $(68 \mathrm{mg}, 0.3 \mathrm{mmol})$ and $\mathbf{2 a}(151 \mathrm{mg}, 0.30 \mathrm{mmol})$ in dried $\mathrm{ACN}(10 \mathrm{~mL})$. The mixture rapidly became a clear solution, which was stirred at ambient temperature for $4 \mathrm{~h}$. Solid $\mathrm{NaHCO}_{3}(800 \mathrm{mg})$ and $\mathrm{H}_{2} \mathrm{O}(0.1 \mathrm{~mL})$ were added sequentially, and the suspension was stirred for an additional $1 \mathrm{~h}$. The inorganic residue was filtered and washed (ACN, $50 \mathrm{~mL}$ ). Volatiles were evaporated from the combined filtrate and wash, and the residue was chromatographed (EtOAc/hexanes, 1:6 $\rightarrow$ EtOAc) to give 3a (157 mg, 78\%). (Stirring for $9 \mathrm{~h}$ at ambient temperature was required for the coupling with 2,3,4,6-tetra- $O$-acetyl- $\beta$-D-glucopyranosyl bromide.)

General Procedure 3: TMSOTf $(0.11 \mathrm{~mL}, 133 \mathrm{mg}, 0.6 \mathrm{mmol})$ was added dropwise to a stirred suspension of $\mathbf{1 b}(68 \mathrm{mg}, 0.3 \mathrm{mmol})$ and $\mathbf{2 a}(151 \mathrm{mg}, 0.3 \mathrm{mmol})$ in dried DCE (5 mL). The resulting clear solution was stirred for $1.5 \mathrm{~h}$ at ambient temperature. Saturated $\mathrm{NaHCO}_{3} / \mathrm{H}_{2} \mathrm{O}(30$ $\mathrm{mL})$ and $\mathrm{CH}_{2} \mathrm{Cl}_{2}(30 \mathrm{~mL})$ were added, and stirring was continued for $10 \mathrm{~min}$. The aqueous layer was separated and extracted with $\mathrm{CH}_{2} \mathrm{Cl}_{2}(30 \mathrm{~mL})$. The combined organic layers were concentrated and added to a column of silica gel. Chromatography (EtOAc/hexanes, 1:3 $\rightarrow$ EtOAc) gave 3a (167 $\mathrm{mg}, 83 \%)$ as single regioisomer.

9-(2,3,5-Tri- $\boldsymbol{O}$-benzoyl- $\boldsymbol{\beta}$-D-ribofuranosyl)-6-(imidazol-1-yl)purine. ${ }^{1} \mathrm{H}$ NMR $\delta 4.72$ (dd, $J=4.3,12.2 \mathrm{~Hz}, 1 \mathrm{H}), 4.86-4.90(\mathrm{~m}, 1 \mathrm{H}), 4.95(\mathrm{dd}, J=3.0,12.3 \mathrm{~Hz}, 1 \mathrm{H}), 6.29(\mathrm{t}, J=5.2 \mathrm{~Hz}, 1 \mathrm{H})$, 6.47-6.50 (m, 2H), $7.24(\mathrm{~s}, 1 \mathrm{H}), 7.35-8.07(\mathrm{~m}, 15 \mathrm{H}), 8.26,8.35,8.65,8.72(4 \times \mathrm{s}, 4 \times 1 \mathrm{H}) ;{ }^{13} \mathrm{C}$ NMR $\delta 62.3,70.3,72.9,79.9,86.4,116.3,122.0,127.2,127.5,128.2,128.7,128.8,129.7,132.4$, 132.8, 132.9, 136.6, 142.2, 144.9, 151.5, 152.1, 164.1, 164.3, 165.0; HMRS Calcd for $\mathrm{C}_{34} \mathrm{H}_{26} \mathrm{~N}_{6} \mathrm{O}_{7} \mathrm{Na}$ : 653.1761, Found: 653.1749 .

\section{9-(2,3,5-Tri- $O$-benzoyl- $\beta$-D-ribofuranosyl)-6-(2-propylimidazol-1-yl)purine (3a). ${ }^{1} \mathrm{H}$} NMR $\delta 1.01(\mathrm{t}, J=7.3 \mathrm{~Hz}, 3 \mathrm{H}), 1.79-1.88(\mathrm{~m}, 2 \mathrm{H}), 3.28(\mathrm{t}, J=7.6 \mathrm{~Hz}, 2 \mathrm{H}), 4.73(\mathrm{dd}, J=4.4,12.2$ $\mathrm{Hz}, 1 \mathrm{H}), 4.86-4.90(\mathrm{~m}, 1 \mathrm{H}), 4.94(\mathrm{dd}, J=2.9,12.2 \mathrm{~Hz}, 1 \mathrm{H}), 6.29(\mathrm{t}, J=5.4 \mathrm{~Hz}, 1 \mathrm{H}), 6.46(\mathrm{t}, J=$ $5.4 \mathrm{~Hz}, 1 \mathrm{H}), 6.50(\mathrm{~d}, J=5.4 \mathrm{~Hz}, 1 \mathrm{H}), 7.10(\mathrm{~d}, J=1.5 \mathrm{~Hz}, 1 \mathrm{H}), 7.36-8.10(\mathrm{~m}, 15 \mathrm{H}), 8.26(\mathrm{~s}, 1 \mathrm{H})$, $8.41(\mathrm{~d}, J=2.0 \mathrm{~Hz}, 1 \mathrm{H}), 8.72(\mathrm{~s}, 1 \mathrm{H}) ;{ }^{13} \mathrm{C}$ NMR $\delta 13.9,21.0,32.3,63.2,71.2,73.7,80.6,87.3$, 120.4, 124.2, 128.1, 128.3-128.5 (ovlp), 129.1, 129.5-129.6 (ovlp), 133.3, 133.6, 133.7, 142.8, 147.6, 150.5, 151.8, 152.8, 165.0, 165.1, 165.9; FAB-MS $m / z 673\left(100 \%, \mathrm{M}+\mathrm{H}^{+}\right)$; HMRS Calcd for $\mathrm{C}_{37} \mathrm{H}_{33} \mathrm{~N}_{6} \mathrm{O}_{7}: 673.2410$, Found: 673.2402 . 
9-(2,3,5-Tri- $O$-benzoyl- $\beta$-D-xylofuranosyl)-6-(2-propylimidazol-1-yl)purine (3b). ${ }^{1} \mathrm{H}$ NMR $\delta 1.01(\mathrm{t}, J=7.3 \mathrm{~Hz}, 3 \mathrm{H}), 1.78-1.87(\mathrm{~m}, 2 \mathrm{H}), 3.27(\mathrm{t}, J=7.8 \mathrm{~Hz}, 2 \mathrm{H}), 4.78(\mathrm{dd}, J=4.9,12.2$ $\mathrm{Hz}, 1 \mathrm{H}), 4.85$ (dd, $J=6.4,12.2 \mathrm{~Hz}, 1 \mathrm{H}), 5.04-5.09$ (m, 1H), 6.01 (d, $J=3.9 \mathrm{~Hz}, 1 \mathrm{H}), 6.31(\mathrm{~s}, 1 \mathrm{H})$, $6.52(\mathrm{~d}, J=2.0 \mathrm{~Hz}, 1 \mathrm{H}), 7.13(\mathrm{~s}, 1 \mathrm{H}), 7.41-8.12(\mathrm{~m}, 15 \mathrm{H}), 8.47,8.52,8.71(3 \times \mathrm{s}, 3 \times 1 \mathrm{H}) ;{ }^{13} \mathrm{C}$ NMR $\delta 13.9,21.0,32.3,61.6,75.2,79.5,79.7,88.4,120.4,123.8,128.0-129.9$ (ovlp), 133.2, 133.89, 133.93, 142.1, 147.5, 150.6, 151.8, 152.7, 164.6, 164.7, 165.8; FAB-MS m/z 695 (100\%, M $+\mathrm{Na}^{+}$); HMRS Calcd for $\mathrm{C}_{37} \mathrm{H}_{32} \mathrm{~N}_{6} \mathrm{O}_{7} \mathrm{Na}$ : 695.2230, Found: 695.2238.

9-(2,3,5-Tri- $O$-benzoyl- $a$-D-arabinofuranosyl)-6-(2-propylimidazol-1-yl)purine $(3 \mathrm{c}) .{ }^{1} \mathrm{H}$ NMR $\delta 1.02(\mathrm{t}, J=7.3 \mathrm{~Hz}, 3 \mathrm{H}), 1.80-1.89(\mathrm{~m}, 2 \mathrm{H}), 3.31(\mathrm{t}, J=7.8 \mathrm{~Hz}, 2 \mathrm{H}), 5.92-5.94(\mathrm{~m}, 2 \mathrm{H})$, $6.56(\mathrm{dd}, J=2.0,2.4 \mathrm{~Hz}, 1 \mathrm{H}), 5.04-5.09(\mathrm{~m}, 1 \mathrm{H}), 6.01$ (d, $J=3.9 \mathrm{~Hz}, 1 \mathrm{H}), 6.31(\mathrm{~s}, 1 \mathrm{H}), 6.52(\mathrm{~d}, J$ $=2.0 \mathrm{~Hz}, 1 \mathrm{H}), 7.13(\mathrm{~s}, 1 \mathrm{H}), 6.58(\mathrm{~d}, J=2.0 \mathrm{~Hz}, 1 \mathrm{H}), 7.13(\mathrm{~d}, J=1.4 \mathrm{~Hz}, 1 \mathrm{H}), 7.37-8.11(\mathrm{~m}, 15 \mathrm{H})$, $8.38(\mathrm{~s}, 1 \mathrm{H}), 8.48(\mathrm{~d}, J=1.0 \mathrm{~Hz}, 1 \mathrm{H}), 8.85(\mathrm{~s}, 1 \mathrm{H}) ;{ }^{13} \mathrm{C} \mathrm{NMR} \delta 13.7,20.8,32.2,63.2,76.9,80.0$, 82.9, 88.9, 120.2, 123.6, 127.8-129.5 (ovlp), 132.9, 133.5, 133.6, 142.4, 147.3, 150.3, 151.5, 152.6, 164.86, 164.90, 165.6; FAB-MS $m / z 695\left(10 \%, \mathrm{M}+\mathrm{Na}^{+}\right), 673\left(100 \%, \mathrm{M}+\mathrm{H}^{+}\right)$; HMRS Calcd for $\mathrm{C}_{37} \mathrm{H}_{32} \mathrm{~N}_{6} \mathrm{O}_{7} \mathrm{Na}$ : 695.2230, Found: 695.2224.

9-(2,3,4,6-Tetra- $O$-acetyl- $\beta$-D-glycopyranosyl)-6-(2-propylimidazol-1-yl)purine $(3 \mathrm{~d}) .{ }^{1} \mathrm{H}$ NMR $\delta 1.02(\mathrm{t}, J=7.3 \mathrm{~Hz}, 3 \mathrm{H}), 1.80(\mathrm{~s}, 3 \mathrm{H}), 1.80-1.89(\mathrm{~m}, 2 \mathrm{H}), 2.06,2.09,2.10(3 \times \mathrm{s}, 3 \times 3 \mathrm{H})$, 3.26-3.36 (m, 2H), $4.08(\mathrm{ddd}, J=2.0,4.9,10.3 \mathrm{~Hz}, 1 \mathrm{H}), 4.18(\mathrm{dd}, J=1.9,12.7 \mathrm{~Hz}, 1 \mathrm{H}), 4.32(\mathrm{dd}, J$ $=4.9,12.7 \mathrm{~Hz}, 1 \mathrm{H}), 5.34(\mathrm{t}, J=9.8 \mathrm{~Hz}, 1 \mathrm{H}), 5.50(\mathrm{t}, J=9.5 \mathrm{~Hz}, 1 \mathrm{H}), 5.70(\mathrm{t}, J=9.5 \mathrm{~Hz}, 1 \mathrm{H}), 6.02$ $(\mathrm{d}, J=9.8 \mathrm{~Hz}, 1 \mathrm{H}), 7.11(\mathrm{~d}, J=1.0 \mathrm{~Hz}, 1 \mathrm{H}), 8.31(\mathrm{~s}, 1 \mathrm{H}), 8.52(\mathrm{~d}, J=1.0 \mathrm{~Hz}, 1 \mathrm{H}), 8.86(\mathrm{~s}, 1 \mathrm{H}) ;{ }^{13} \mathrm{C}$ NMR $\delta 13.8,19.9,20.26,20.32,20.4,20.9,32.4,61.3,67.5,69.9,72.5,74.8,80.2,120.3,123.0$, 128.3 141.6, 147.6, 150.5, 151.9, 153.1, 168.8, 169.2, 169.6; FAB-MS $m / z 581\left(10 \%, \mathrm{M}+\mathrm{Na}^{+}\right)$, $559\left(100 \%, \mathrm{M}+\mathrm{H}^{+}\right)$; HMRS Calcd for $\mathrm{C}_{25} \mathrm{H}_{30} \mathrm{~N}_{6} \mathrm{O}_{9} \mathrm{Na}: 581.1972$, Found: 581.1973.

Synthesis of 6-Methoxypurine Nucleosides 5. General Procedure 4: Dowex $1 \times 2\left(\mathrm{OH}^{-}\right)$ resin $(10 \mathrm{~mL})$ was added to a solution of $\mathbf{3 a}(1.5 \mathrm{~g}, 2.23 \mathrm{mmol})$ in $\mathrm{MeOH}$, and the suspension was stirred overnight at ambient temperature. The resin was filtered and thoroughly washed $(\mathrm{MeOH}, 10$ $\times 20 \mathrm{~mL}$ ). Volatiles were evaporated from the combined filtrates, and chromatography of the residue $\left(\right.$ EtOAc $\rightarrow \mathrm{MeOH} /$ EtOAc, 1:10) gave 6-methoxy-9-( $\beta$-D-ribofuranosyl)purine ${ }^{3}$ (5a), which was recrystallized $(\mathrm{EtOH})$ to provide a clean sample of 5a: mp $142-143{ }^{\circ} \mathrm{C}$; UV $(\mathrm{MeOH}) \max 248$ $\mathrm{nm}(\varepsilon 12200), \min 222 \mathrm{~nm}\left(\varepsilon\right.$ 3600); ${ }^{1} \mathrm{H}$ NMR $\left(\mathrm{CD}_{3} \mathrm{OD}\right) \delta 3.78(\mathrm{dd}, J=3.4,12.7 \mathrm{~Hz}, 1 \mathrm{H}), 3.88$ (dd,

\footnotetext{
${ }^{3}$ Johnson, J. A., Jr.; Thomas, H. J.; Schaeffer, H. J. J. Am. Chem. Soc. 1958, 80, 699-702.
} 
$J=2.9,12.2 \mathrm{~Hz}, 1 \mathrm{H}), 4.16-4.19(\mathrm{~m}, 1 \mathrm{H}), 4.19(\mathrm{~s}, 3 \mathrm{H}), 4.34-4.37(\mathrm{~m}, 1 \mathrm{H}), 4.74(\mathrm{t}, J=5.6 \mathrm{~Hz}, 1 \mathrm{H})$, $6.07(\mathrm{~d}, J=5.9 \mathrm{~Hz}, 1 \mathrm{H}), 8.51,8.54(2 \times \mathrm{s}, 2 \times 1 \mathrm{H}) ;{ }^{13} \mathrm{C} \mathrm{NMR}\left(\mathrm{DMSO}-d_{6}\right) \delta 54.0,61.3,70.4,73.8$, 85.7, 87.8, 121.2, 142.5, 151.72, 151.76, 160.4; FAB-MS m/z 305 (70\%, M + Na $), 319$ (100\%); HMRS Calcd for $\mathrm{C}_{11} \mathrm{H}_{14} \mathrm{~N}_{4} \mathrm{O}_{5} \mathrm{Na}$ : 305.0862, Found: 305.0876.

6-Methoxy-9-( $\beta$-D-xylofuranosyl)purine (5b). Recrystallized from $\mathrm{MeOH} / \mathrm{EtOAc:} \mathrm{mp}$ 157-160 ${ }^{\circ} \mathrm{C}$; UV (MeOH) $\max 248 \mathrm{~nm}(\varepsilon 11800)$, min $222 \mathrm{~nm}\left(\varepsilon\right.$ 3800); ${ }^{1} \mathrm{H}$ NMR $\left(\mathrm{CD}_{3} \mathrm{OD}\right) \delta 3.94$ $(\mathrm{dd}, J=5.9,11.7 \mathrm{~Hz}, 1 \mathrm{H}), 3.97(\mathrm{dd}, J=4.9,11.7 \mathrm{~Hz}, 1 \mathrm{H}), 4.22(\mathrm{dd}, J=1.5,3.9 \mathrm{~Hz}, 1 \mathrm{H}), 4.18(\mathrm{~s}$, $3 \mathrm{H}), 4.36-4.39(\mathrm{~m}, 1 \mathrm{H}), 4.46(\mathrm{t}, J=1.7 \mathrm{~Hz}, 1 \mathrm{H}), 6.10(\mathrm{~d}, J=1.5 \mathrm{~Hz}, 1 \mathrm{H}), 8.52,8.53(2 \times \mathrm{s}, 2 \times$ $1 \mathrm{H}) ;{ }^{13} \mathrm{C}$ NMR $\left(\mathrm{CD}_{3} \mathrm{OD}\right) \delta 55.0,61.6,77.2,82.7,85.3,92.3,122.3,143.9,152.4,153.3,162.3$; FAB-MS m/z $282\left(3 \%, \mathrm{M}^{+}\right), 151(100 \%)$; HMRS Calcd for $\mathrm{C}_{11} \mathrm{H}_{14} \mathrm{~N}_{4} \mathrm{O}_{5}: 282.0964$, Found: 282.0996.

9-(a-D-Arabinofuranosyl)-6-methoxypurine ${ }^{4}(\mathbf{5 c}) .{ }^{1} \mathrm{H}$ NMR $\left(\mathrm{CD}_{3} \mathrm{OD}\right) \delta 3.74(\mathrm{dd}, J=4.9$, $11.8 \mathrm{~Hz}, 1 \mathrm{H}), 3.80(\mathrm{dd}, J=3.9,11.7 \mathrm{~Hz}, 1 \mathrm{H}), 4.18(\mathrm{~s}, 3 \mathrm{H}), 4.21(\mathrm{t}, J=5.1 \mathrm{~Hz}, 1 \mathrm{H}), 4.39-4.43(\mathrm{~m}$, $1 \mathrm{H}), 4.46(\mathrm{t}, J=4.2 \mathrm{~Hz}, 1 \mathrm{H}), 6.10(\mathrm{~d}, J=3.9 \mathrm{~Hz}, 1 \mathrm{H}), 8.47,8.53(2 \times \mathrm{s}, 2 \times 1 \mathrm{H}) ;{ }^{13} \mathrm{C} \mathrm{NMR}$ $\left(\mathrm{CD}_{3} \mathrm{OD}\right) \delta$ 55.0, 62.9, 77.4, 81.7, 88.3, 91.9, 122.6, 143.8, 152.9, 153.5, 162.4; FAB-MS $m / z .283$ $\left(100 \%, \mathrm{M}+\mathrm{H}^{+}\right)$; HMRS Calcd for $\mathrm{C}_{11} \mathrm{H}_{15} \mathrm{~N}_{4} \mathrm{O}_{5}: 283.1042$, Found: 283.1043 .

9-( $\beta$-D-Glucopyranosyl)-6-methoxypurine (5d). Recrystallized from MeOH: mp 188-190 ${ }^{\circ} \mathrm{C}$; UV (MeOH) $\max 247 \mathrm{~nm}\left(\varepsilon 10\right.$ 900), $\min 218 \mathrm{~nm}(\varepsilon 2500) ;{ }^{1} \mathrm{H}$ NMR $\left(\mathrm{CD}_{3} \mathrm{OD}\right) \delta 3.54-5.62(\mathrm{~m}$, 3H), $3.74(\mathrm{dd}, J=5.4,12.2 \mathrm{~Hz}, 1 \mathrm{H}), 3.89$ (dd, $J=2.0,12.2 \mathrm{~Hz}, 1 \mathrm{H}), 4.09-4.13$ (m, 1H), 4.19 (s, $3 \mathrm{H}), 5.66(\mathrm{~d}, J=9.3 \mathrm{~Hz}, 1 \mathrm{H}), 8.50,8.53(2 \times \mathrm{s}, 2 \times 1 \mathrm{H}) ;{ }^{13} \mathrm{C} \mathrm{NMR}\left(\mathrm{CD}_{3} \mathrm{OD}\right) \delta 54.0,60.9,69.8$, 71.3, 77.1, 80.2, 83.3, 120.7, 142.7, 151.7, 152.3, 160.3; FAB-MS m/z $313\left(100 \%, \mathrm{M}^{+} \mathrm{H}^{+}\right)$; HMRS Calcd for $\mathrm{C}_{12} \mathrm{H}_{17} \mathrm{~N}_{4} \mathrm{O}_{6}: 313.1148$, Found: 313.1143 .

Deacylation of Compounds 3. General Procedure 5: A solution of 3a (900 mg, 1.34 mmol) in $\mathrm{NH}_{3} / \mathrm{MeOH}(20 \mathrm{~mL})$ was stirred overnight at ambient temperature. Volatiles were evaporated, and the residue was chromatographed (EtOAc $\rightarrow \mathrm{MeOH} / \mathrm{EtOAc}, 1: 10)$ to give 9-( $\beta$-Dribofuranosyl)-6-(2-propylimidazol-1-yl)purine (6a), which was recrystallized (MeOH) to provide a clean sample of 6a: $\operatorname{mp} 213-215^{\circ} \mathrm{C}$; UV (MeOH) max $278 \mathrm{~nm}(\varepsilon 14700)$, min $236 \mathrm{~nm}(\varepsilon$ 3600); ${ }^{1} \mathrm{H}$ NMR $\left(\mathrm{CD}_{3} \mathrm{OD}\right) \delta 0.96(\mathrm{t}, J=7.3 \mathrm{~Hz}, 3 \mathrm{H}), 1.71-1.79(\mathrm{~m}, 2 \mathrm{H}), 3.26(\mathrm{t}, J=7.3 \mathrm{~Hz}, 2 \mathrm{H}), 3.80$, $3.91(2 \times \mathrm{dd}, J=2.9,12.2 \mathrm{~Hz}, 2 \times 1 \mathrm{H}), 4.17-4.20(\mathrm{~m}, 1 \mathrm{H}), 4.39(\mathrm{t}, J=4.4 \mathrm{~Hz}, 1 \mathrm{H}), 4.76(\mathrm{t}, J=5.4$

\footnotetext{
${ }^{4}$ Jones, L. A.; Moorman, A. R.; Chamberlain, S. D.; de Miranda, P.; Reynolds, D. J.; Burns, C. L.; Krenitsky, T. A. J. Med. Chem. 1992, 35, 56-63.
} 
$\mathrm{Hz}, 1 \mathrm{H}), 6.19(\mathrm{~d}, J=5.4 \mathrm{~Hz}, 1 \mathrm{H}), 7.07,8.40,8.79,8.86(4 \times \mathrm{s}, 4 \times 1 \mathrm{H}) ;{ }^{13} \mathrm{C} \mathrm{NMR}\left(\mathrm{CD}_{3} \mathrm{OD}\right) \delta 14.3$, 22.7, 32.8, 63.0, 72.3, 76.1, 87.7, 90.9, 122.4, 126.2 , 128.4, 146.5, 148.6, 152.0, 152.8, 154.9; FABMS $m / z 383\left(100 \%, \mathrm{M}+\mathrm{Na}^{+}\right)$; HMRS Calcd for $\mathrm{C}_{16} \mathrm{H}_{20} \mathrm{~N}_{6} \mathrm{O}_{4} \mathrm{Na}: 383.1444$, Found: 383.1450 .

9-( $\beta$-D-Xylofuranosyl)-6-(2-propylimidazol-1-yl)purine (6b). Recrystallized from MeOH/EtOAc: mp 149-151 ${ }^{\circ} \mathrm{C}$; UV (MeOH) max $278 \mathrm{~nm}$ (ع 14900$)$, min $237 \mathrm{~nm}\left(\varepsilon\right.$ 3800); ${ }^{1} \mathrm{H}$ NMR $\left(\mathrm{CD}_{3} \mathrm{OD}\right) \delta 0.96(\mathrm{t}, J=7.3 \mathrm{~Hz}, 3 \mathrm{H}), 1.71-1.79(\mathrm{~m}, 2 \mathrm{H}), 3.27(\mathrm{t}, J=7.3 \mathrm{~Hz}, 2 \mathrm{H}), 3.98(\mathrm{dd}, J=$ 5.9, $12.2 \mathrm{~Hz}, 1 \mathrm{H}), 4.00(\mathrm{dd}, J=4.9,12.2 \mathrm{~Hz}, 1 \mathrm{H}), 4.24-4.26(\mathrm{~m}, 1 \mathrm{H}), 4.41-4.44(\mathrm{~m}, 1 \mathrm{H})$, 4.49-4.50 (m, 1H), 6.23 (d, $J=1.5 \mathrm{~Hz}, 1 \mathrm{H}), 7.07$ (d, $J=2.0 \mathrm{~Hz}, 1 \mathrm{H}), 8.38$ (d, $J=1.5 \mathrm{~Hz}, 1 \mathrm{H}), 8.76$, $8.88(2 \times \mathrm{s}, 2 \times 1 \mathrm{H}) ;{ }^{13} \mathrm{C} \mathrm{NMR}\left(\mathrm{CD}_{3} \mathrm{OD}\right) \delta 14.3,22.7,32.8,61.6,77.0,82.7,85.6,92.2,122.4$, 125.7, 128.3, 146.5, 148.2, 152.0, 152.9, 154.7; FAB-MS $m / z, 361\left(100 \%, \mathrm{M}+\mathrm{H}^{+}\right)$; HMRS Calcd for $\mathrm{C}_{16} \mathrm{H}_{21} \mathrm{~N}_{6} \mathrm{O}_{4}$ : 361.1624, Found: 361.1631 .

9-(a-D-Arabinofuranosyl)-6-(2-propylimidazol-1-yl)purine (6c). Recrystallized from

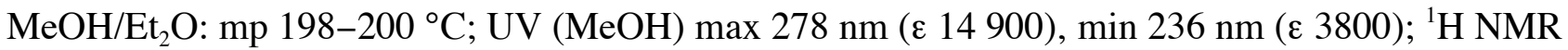
$\left(\mathrm{DMSO}-d_{6}\right) \delta 0.93(\mathrm{t}, J=7.3 \mathrm{~Hz}, 3 \mathrm{H}), 1.69-1.77(\mathrm{~m}, 2 \mathrm{H}), 3.19(\mathrm{t}, J=7.3 \mathrm{~Hz}, 2 \mathrm{H}), 3.55-3.68(\mathrm{~m}$, 2H), 4.08-4.11 (m, 1H), 4.29-4.33 (m, 1H), 4.73-4.76 (m, 1H), 5.00 (t, J = 5.4 Hz, 1H), 5.66 (d, $J$ $=5.4 \mathrm{~Hz}, 1 \mathrm{H}), 5.92(\mathrm{~d}, J=5.4 \mathrm{~Hz}, 1 \mathrm{H}), 6.09(\mathrm{~d}, J=4.4 \mathrm{~Hz}, 1 \mathrm{H}), 7.07,8.36,8.92,8.95(4 \times \mathrm{s}, 4 \times$ $1 \mathrm{H}) ;{ }^{13} \mathrm{C}$ NMR (DMSO- $\left.d_{6}\right) \delta 13.9,20.8,31.6,61.1,75.0,79.3,86.0,89.1,120.7,123.9,128.0$, 145.5, 146.7, 149.2, 151.6, 153.1; FAB-MS m/z $361\left(100 \%, \mathrm{M}+\mathrm{H}^{+}\right)$; HMRS Calcd for $\mathrm{C}_{16} \mathrm{H}_{21} \mathrm{~N}_{6} \mathrm{O}_{4}$ : 361.1624, Found: 361.1630.

9-( $\beta$-D-Glycopyranosyl)-6-(2-propylimidazol-1-yl)purine (6d). Recrystallized from $\mathrm{EtOH} / \mathrm{Et}_{2} \mathrm{O}: \mathrm{mp} 166-168{ }^{\circ} \mathrm{C} ; \mathrm{UV}(\mathrm{MeOH}) \max 277 \mathrm{~nm}(\varepsilon 14800), \min 233 \mathrm{~nm}\left(\varepsilon\right.$ 4100); ${ }^{1} \mathrm{H} \mathrm{NMR}$ $\left(\mathrm{CD}_{3} \mathrm{OD}\right) \delta 0.96(\mathrm{t}, J=7.3 \mathrm{~Hz}, 3 \mathrm{H}), 1.71-1.79(\mathrm{~m}, 2 \mathrm{H}), 3.27(\mathrm{t}, J=7.8 \mathrm{~Hz}, 2 \mathrm{H}), 3.56-3.66(\mathrm{~m}, 2 \mathrm{H})$, $3.75(\mathrm{dd}, J=5.4,12.2 \mathrm{~Hz}, 1 \mathrm{H}), 3.90(\mathrm{dd}, J=2.0,10.7 \mathrm{~Hz}, 1 \mathrm{H}), 4.17$ (t, $J=9.3 \mathrm{~Hz}, 1 \mathrm{H}), 5.77(\mathrm{~d}, J=$ $9.3 \mathrm{~Hz}, 2 \mathrm{H}), 7.09,8.39,8.73,8.89(4 \times \mathrm{s}, 4 \times 1 \mathrm{H}) ;{ }^{13} \mathrm{C}$ NMR $\left(\mathrm{DMSO}-d_{6}\right) \delta 13.9,20.8,31.5,61.0$, 69.8, 71.4, 77.1, 80.3, 83.4, 120.7, 123.5, 128.0, 145.6, 146.7, 149.3, 151.7, 153.7; FAB-MS m/z $391\left(55 \%, \mathrm{M}+\mathrm{Na}^{+}\right), 237(100 \%)$; HMRS Calcd for $\mathrm{C}_{17} \mathrm{H}_{23} \mathrm{~N}_{6} \mathrm{O}_{5}: 391.1729$, Found: 391.1721 . 

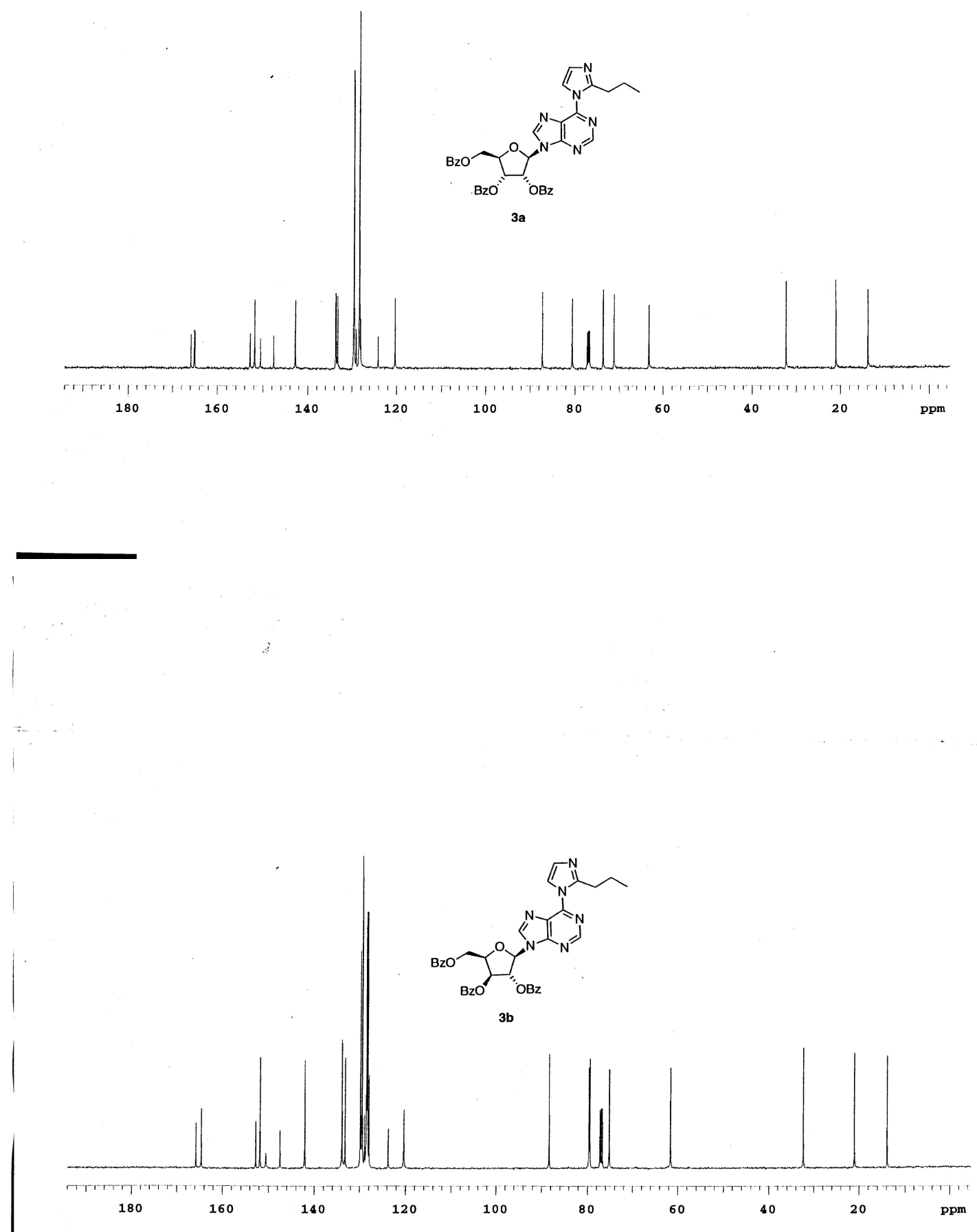

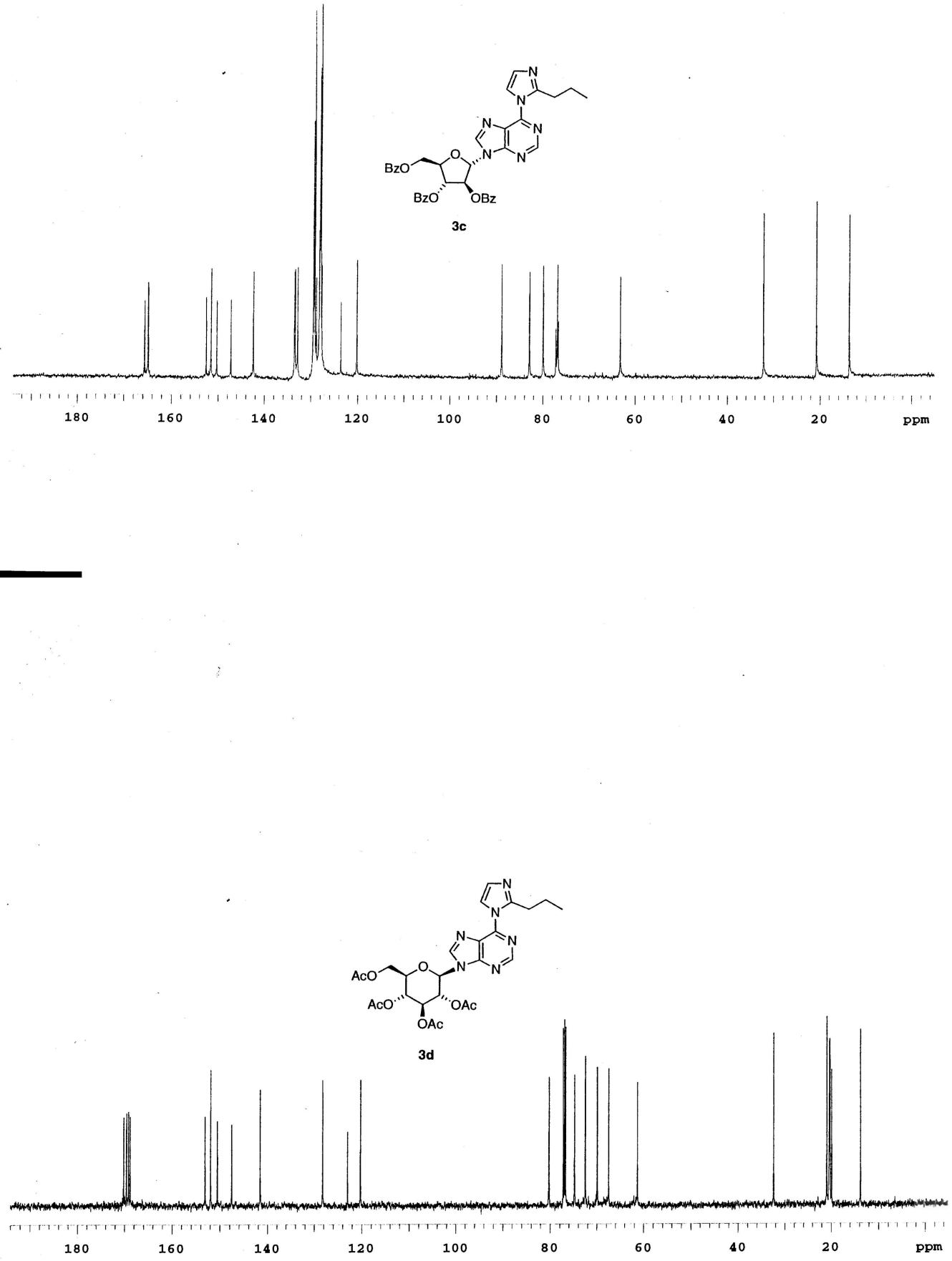

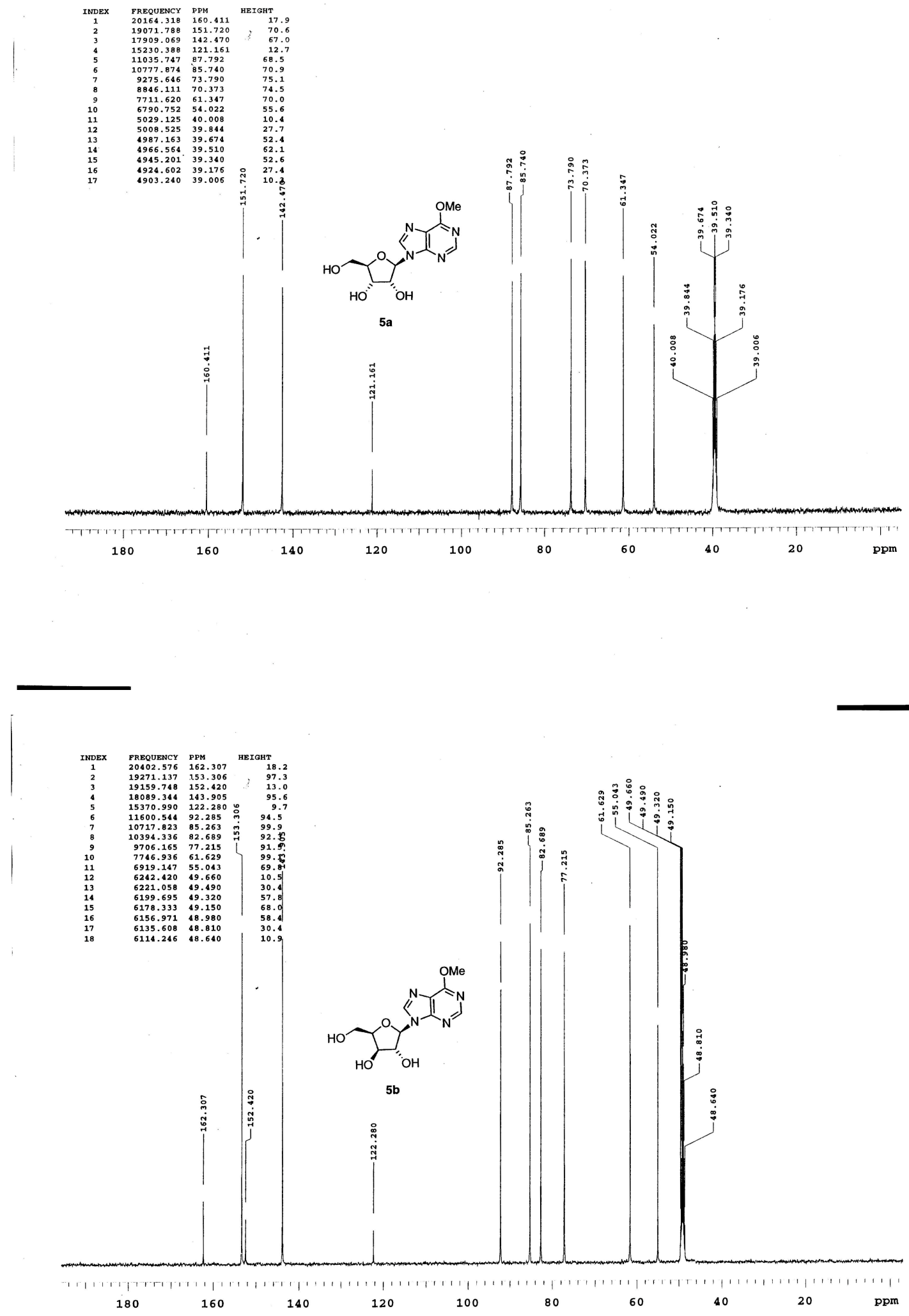

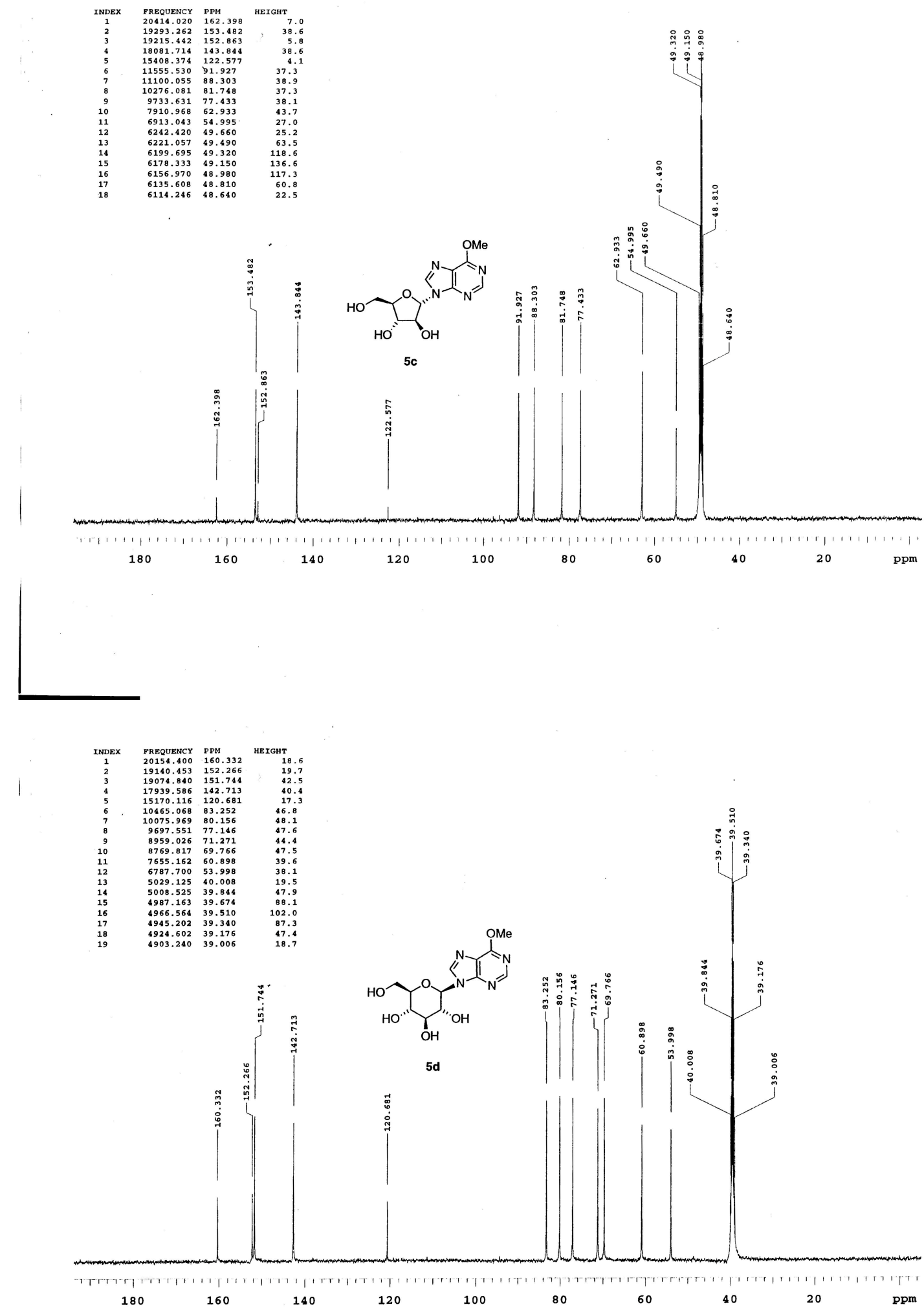

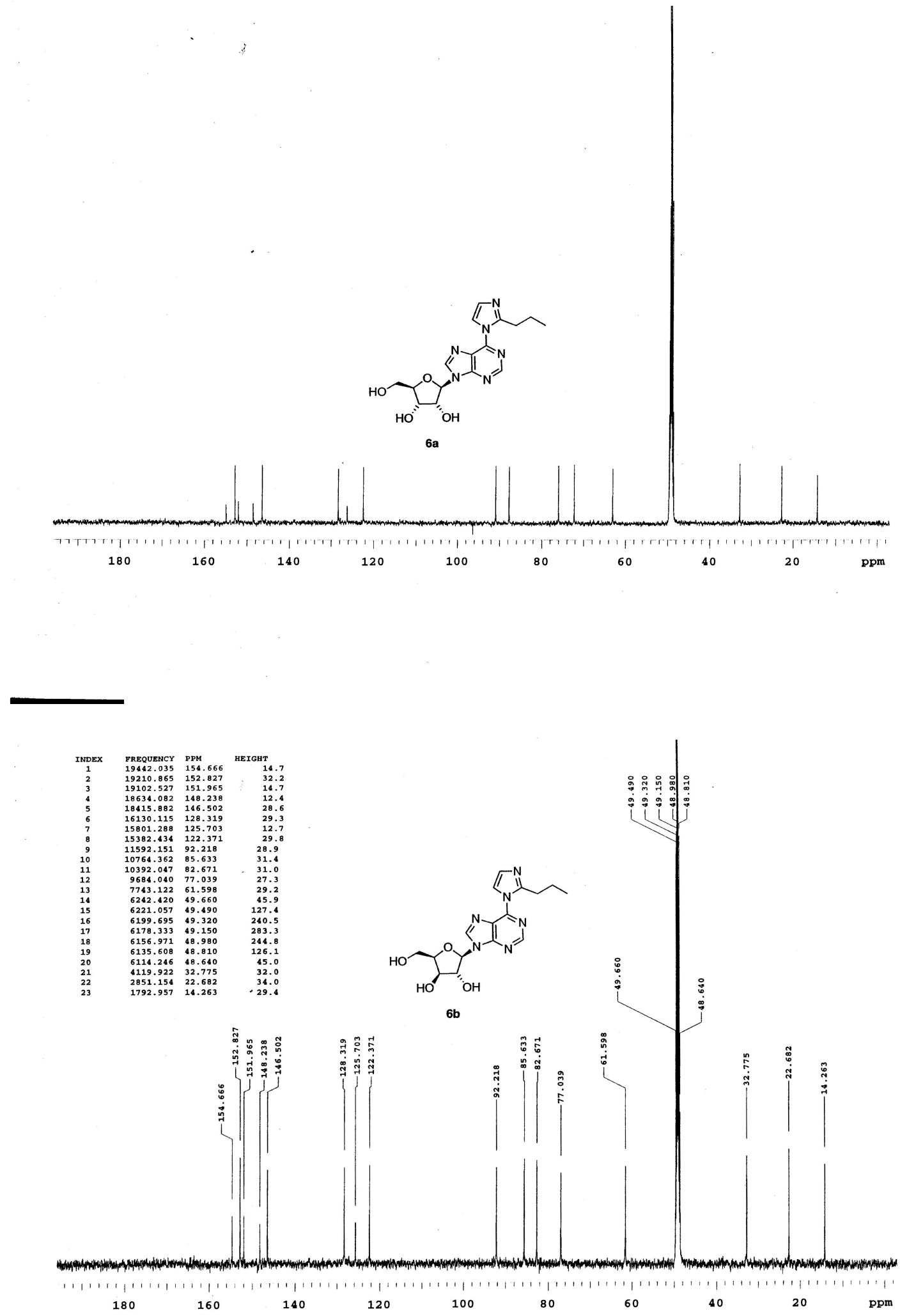

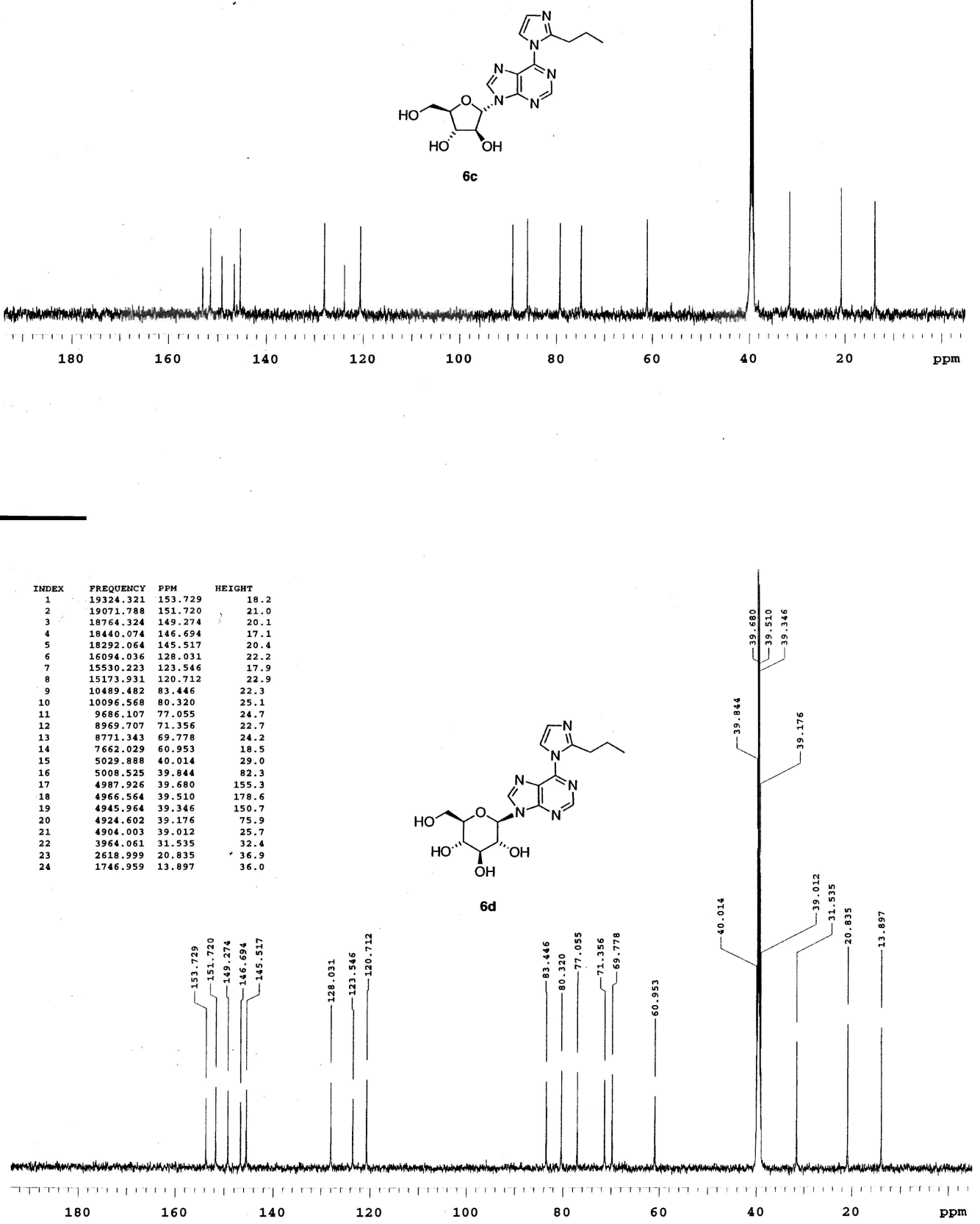